\title{
ABSTRACTS IN ECHOCARDIOGRAPHY 2000
}

Pulse-inversion myocardial contrast echocardiography (MCE) identifies adenosine induced acoustic defects in the myocardium of patients with critical coronary stenoses

D T Chin, J C Cooke, J Hancock, M J Monaghan; King's College Hospital, London

Harmonic MCE detects changes in myocardial blood flow and volume during stress induced ischaemia. These changes are difficult to visualise and their detection requires complex data post processing. Pulse inversion (PI) imaging is a new technology that amplifies contrast signal-to-noise ratio and may magnify acoustic changes during myocardial ischaemia. We therefore assessed the acoustic intensity (AI) of images produced by the technique before/during vasodilator stress.

Methods: 19 subjects with no previous infarction, 10 with lone angiographic LAD stenoses $>80 \%, 2$ with lone moderate LAD stenoses and 7 with RCA and circumflex but no LAD disease, were studied. The coronary supply to each echocardiographic segment was graded. MCE was performed with the transpulmonary contrast agent Sonazoid (Nycomed-Amersham) and end-systolic triggered PI imaging on an ATL HDI-5000cv system. Linear myocardial and left ventricular (LV) AI was measured from digital loops of images using HDI-lab (ATL) software. \% change in mean myocardial AI before and during adenosine was calculated with $(\% \mathrm{~N})$ and without $(\% \mathrm{~A})$ normalisation for LV AI. Qualitative analysis of myocardial AI and geometry was performed.

Results: Data from 26 apical views, $71 \mathrm{mid}$ and apical segments and $26 \mathrm{LV}$ regions of interest showed adenosine induced falls in myocardial AI were higher in the territory of vessels with critical stenoses. LV AI before and during adenosine remained unchanged. Visual analysis showed inducible acoustic defects in $8 / 15$ ischaemic and $0 / 12$ non-ischaemic apical segments $(\mathrm{p}=0.003)$

Conclusions: PI imaging detects $20-30 \%$ falls in AI during ischaemia that are sufficient in some cases to allow visual detection of inducible defects in nearfield myocardium. PI technology may therefore have the potential for real time visual assessment of myocardial ischaemia.

\begin{tabular}{llll}
\hline & No segments & $\% A(+/-s d)$ & $\% N(+/$-sd $)$ \\
\hline Stenosis & 32 & $31.0(26)$ & $22.4(31)$ \\
No stenosis & 39 & $9.0(39)$ & $6.5(39)$ \\
P value & & 0.008 & 0.062 \\
\hline
\end{tabular}

\section{Atrioventricular plane displacement changes during} pregnancy

N Kametas, ${ }^{1}$ F McAuliffe, ${ }^{1}$ J Hancock, ${ }^{2}$ J Chambers, ${ }^{3}$ K N Nicolaides ${ }^{1}$ ${ }^{1}$ Harris Birthright Research Centre for Fetal Medicine and ${ }^{2}$ Cardiac Department, King's College Hospital, London; ${ }^{3}$ Cardiac Department, St Thomas' Hospital, London

The measurement of the atrioventricular plane displacement (AVPD) may provide a sensitive sign of early changes in left ventricular (LV) function. The aim of this study was to establish the normal changes of the AVPD during pregnancy. In a cross sectional study, nineteen nonpregnant women (mean age 32 years) and 132 pregnant women with singleton pregnancies (mean age 31 years) underwent $M$-mode echocardiography and blood pressure measurements. The parasternal long axis view was used for standard M-mode measurements of the LV and the apical 4 and 2 chamber view for the measurement of the AVPD and activation time (AT) (the time from the $\mathrm{Q}$ wave of the ECG to the initiation of the AVPD) at the lateral, septal, anterior, and inferior planes respectively.

Results: Fractional Shortening (FS) and Ejection Fraction (EF) remained constant up to about 30 weeks of gestation and subsequently fell below prepregnancy levels $(\mathrm{p}<0.01)$. Left ventricular AVPD increased with gestation by about $10 \%$ in all four regions, to reach a peak at about 23 weeks, with a subsequent reduction at term to levels $14 \%$ lower than those of the non-pregnant controls $(p<0.0001)$. Activation Time (AT) fell from early first trimester to a nadir at about 20 weeks to levels $15 \%$ lower than the non-pregnant values and subsequently increased towards term to levels $25 \%$ higher than non-pregnant controls $(\mathrm{p}<0.001)$. Mean Arterial Pressure (MAP) fell from early first trimester to a nadir at about 20 weeks to levels $4 \%$ lower than the non-pregnant values and subsequently increased towards term to levels $5 \%$ higher than non-pregnant controls $(p<0.001)$. The mean values in early pregnancy $(10-14$ weeks) were significantly lower than non-pregnant controls for the MAP and Activation Time but not for the AVPD, EF, or FS.

Conclusion: Pregnancy is associated with an initial decrease and a subsequent increase in Mean Arterial Pressure. The atrioventricular plane displacement and activation time measurements reflect better the changes in Mean Arterial Pressure than do conventional measurements of systolic function.

\section{Structural changes of the left ventricle during pregnancy and} left ventricular wall stress

N Kametas, ${ }^{1}$ F McAuliffe,,${ }^{1}$ J Hancock, ${ }^{2}$ J Chambers,${ }^{3} \mathrm{~K}$ N Nicolaides ${ }^{1}$ ${ }^{1}$ Harris Birthright Research Centre for Fetal Medicine and ${ }^{2}$ Cardiac Department, King's College Hospital, London; ${ }^{3}$ Cardiac Department, St. Thomas' Hospital, London

Myocardial remodelling is an adaptive mechanism in situations of increased haemodynamic load, such as pregnancy, when it has been postulated that maternal haemodynamics may play an important role in complications such as pre-eclampsia. There is conflicting information regarding structural changes in left ventricular (LV) in normal pregnancy. In a cross-sectional study, 19 non-pregnant and 132 pregnant women at different stages of pregnancy underwent M-mode echocardiography from the parasternal long axis view and blood pressure (BP) measurements with an automated device (Spacelabs 90207). Univariate regression analysis and Student's $t$ test were used to examine changes with gestation and the differences between non-pregnant and pregnant women at different trimesters of pregnancy; the $\mathrm{p}$ values of the regression analyses and $t$ tests are given for each parameter.

Results: Left ventricular end diastolic diameter (LVIDd) increased from early pregnancy to a peak at about 30 weeks $(14 \%$ above non-pregnant levels) then remaining stable to term $(p=0.001)$. LV end systolic diameter (LVIDs), LV posterior wall (LVPW) and intraventricular septum (IVS) dimensions in systole and diastole increased linearly with gestation (LVIDs, IVSd, IVSs: $p<0.0001$, LVPWd, LVPWs: $p=0.003$ ) but there was no difference between nonpregnant controls and early pregnancy (10-14 weeks). Systolic BP increased with gestation to levels $4 \%$ higher at term than non-pregnant controls $(p=0.02)$. Diastolic BP decreased in early pregnancy, reaching a nadir at about 20 weeks at levels $8 \%$ lower than non-pregnant controls and thereafter increased at term to levels $7 \%$ higher than non-pregnant controls $(p<0.0001)$. LV Meridian Wall Stress increased with gestation $(p=0.009)$. Early pregnancy levels (10-14 weeks) were not different than non-pregnant controls. Conclusion: the increase in heart size mirrors the changes in preload and afterload known to occur during pregnancy.

The effect of harmonic imaging on measurement of cyclic variation in intergrated backscatter

J C Cooke, J Hancock, M J Monaghan; King's College Hospital, London

Second harmonic imaging (2HI) was developed to enhance the detection of contrast microspheres. This technology has led to an increased ability to define the endocardial border and possibly to detect perfusion within the myocardium. However, even in the absence of contrast, it has been found that harmonic imaging enhances endocardial definition. As a result, it has become routine software on echo machines. Ultrasonic integrated backscatter (IBS) is another new ultrasound technology. This demonstrates total reflected ultrasound from the myocardium. It has been shown to vary in a cyclical fashion with the cardiac cycle. Cyclic IBS is blunted following myocardial infarction (MI) in myocardial segments supplied by the infarct related artery (IRA). It is possible 
that $2 \mathrm{HI}$ imaging may increase the application of IBS in patients with poor echo windows. However, the effect of $2 \mathrm{HI}$ on the measurement of IBS is not known. In the present study, we measured cyclic IBS in segments of the myocardium supplied by the IRA as well as distant segments both in fundamental mode and $2 \mathrm{HI}$ in 30 patients following MI, keeping all system settings the same throughout.

Results: In fundamental mode $85 \%$ of segments could be visualised and in $2 \mathrm{HI}$ mode $90 \%$.

Conclusions: Cyclic IBS can be measured in $2 \mathrm{HI}$ mode and can be used interchangeably with fundmental imaging. Because it is easier to define the myocardial borders with this technology, this measurement may be more accurate, particularly in patients with difficult echo windows.

\begin{tabular}{lllllll}
\hline View & $\begin{array}{l}\text { Fund } \\
\text { IRA }\end{array}$ & 2HI IRA & p value & $\begin{array}{l}\text { Fund } \\
\text { normal }\end{array}$ & $\begin{array}{l}\text { 2HI } \\
\text { normal }\end{array}$ & p value \\
\hline PSAX & $3.45 \mathrm{~dB}$ & $4.11 \mathrm{~dB}$ & $\mathrm{~ns}$ & $5.50 \mathrm{~dB}$ & $6.30 \mathrm{~dB}$ & $\mathrm{~ns}$ \\
PLAX & $3.87 \mathrm{~dB}$ & $4.71 \mathrm{~dB}$ & $\mathrm{~ns}$ & $5.65 \mathrm{~dB}$ & $6.65 \mathrm{~dB}$ & $\mathrm{~ns}$ \\
AP4C & $3.46 \mathrm{~dB}$ & $3.93 \mathrm{~dB}$ & $\mathrm{~ns}$ & $4.70 \mathrm{~dB}$ & $5.60 \mathrm{~dB}$ & $\mathrm{~ns}$ \\
AP2C & $3.40 \mathrm{~dB}$ & $3.56 \mathrm{~dB}$ & $\mathrm{~ns}$ & $5.70 \mathrm{~dB}$ & $6.10 \mathrm{~dB}$ & $\mathrm{~ns}$ \\
\hline
\end{tabular}

\section{The holy grail?-real time myocardial perfusion imaging}

J Hancock, J C Cooke, M J Monaghan; King's College Hospital, London

Development of ultrasound technology and intravenous (IV) contrast agents makes sensitive detection of myocardial perfusion a real possibility. Because the high insonsating energy required for successful imaging using second harmonic and harmonic power Doppler destroys contrast microspheres, this has been difficult to achieve in humans unless intermittent imaging is used. Intermittent imaging, however, precludes acquisition of simultaneous wall motion information. Recently, new highly sensitive ultrasound technology has been developed which makes real time perfusion imaging (RTPI) a possibility using much lower, non-destructive insonating energy. In this study we set out to determine the feasibility of RTPI in humans using 2 new technologies. RTPI was performed in 16 patients using either an Agilent Sonos 5500 (pulse modulation imaging) or an ATL HDI 5000 (power pulse inversion). Slow bolus injections or infusions of IV Optison were administered. The 4 standard echo views were utilised, although all views were not recorded in every patient. The images were analysed using a standard wall motion scoring system and a perfusion score of $2=$ homogeneous uptake, $1=$ patchy uptake, $0=$ no uptake. The presence of artefacts precluding assessment was also reported.

Results: Of a possible 278 segments, 176 segments had homogeneous contrast uptake, 22 had patchy uptake, and 80 segments $(28 \%)$ were subject to artefacts and attentuation precluding perfusion assessment. The table below shows the \% of myocardial segments which were opacified with contrast.

Myocardial opacification was more difficult to evaluate in segments distant from the transducer and at the edges of the scan plane. Of the hypokinetic and normokinetic segments in which we would expect normal perfusion, $99.4 \%$ demonstrated homogeneous contrast uptake. In comparison, $70 \%$ of the akinetic segments had patchy uptake only $(\mathrm{p}<0.0001)$.

Conclusions: Although there are some technical limitations, RTPI is a significant advance in contrast echo facilitating simultaneous perfusion and wall motion assessment.

\begin{tabular}{llllll}
\hline & Septal & Anterior & Apical & Lateral & Inf-Post \\
\hline Parasternal & $87 \%$ & $100 \%$ & N/A & $8 \%$ & $27 \%$ \\
Apical & $97 \%$ & $25 \%$ & $98 \%$ & $50 \%$ & $96 \%$ \\
\hline
\end{tabular}

Do false aneurysms following aortic root surgery necessitate surgery?

N R A Clarke, A D Kelion, A P Banning; Cardiology Department, John Radcliffe Hospital, Oxford

Introduction: False aneurysm (FA) is a well recognised complication of aortic root surgery, but little is known of its natural history with conservative management. We investigated the outcome of patients with FA followed up by transoesophageal echocardiography (TOE). Methods: All patients undergoing TOE in our centre for FA 1996-2000 were identified. The case notes and serial TOE studies (intervals of 2-6 months) were reviewed to provide outcome data. FA was diagnosed on the basis of a periannular or periaortic echo free space with colour flow entering the space.

Results: 7 patients with FA were identified (mean size $6.4+/-1.7 \mathrm{~cm}$ ). Systolic compression of the aortic root was present in 5 patients. 6 FAs followed emergency operations, 5 of which were for aortic root abscess ( 2 native and 3 prosthetic endocarditis). FA was diagnosed a mean of 12 months after surgery (range 1-60 months). 1 patient had signs of SVC compression, 2 had severe aortic regurgitation, and 4 were identified on routine transthoracic echocardiography. Only 1 patient underwent further surgery (for SVC obstruction), and died postoperatively. 6 patients were managed conservatively and were followed up for $18+/-5$ months. There were 2 deaths, one from heart failure and the other from myocardial infarction. No patient suffered FA rupture.

Discussion: The majority of FAs follow emergency surgery for aortic root abscess. FA rupture is not a significant danger, in the medium term. Further intervention should be considered only where other cardiac complications have developed.

\section{Intraoperative transoesophageal echocardiography: the role of the sonographer}

J Hayton, R Keal, L Ricketts, S Waterfield; Departments of Echocardiography, Glenfield Hospital, Groby Road, Leicester

Introduction: Intraoperative transoesophageal echocardiography is increasingly requested by cardiothoracic surgeons to evaluate the heart during surgery. It is normally performed by cardiologists, radiologists, or cardiac anaesthetists. However, in the UK, anaesthetists have little or no formal basic echocardiography training. The American Society of Anaesthesiologists (ASA) has produced TOE practice guidelines, but extensive training is required to fulfil them. In our institution, a busy cardiothoracic tertiary referral centre, we have adopted a different strategy.

Purpose: In the UK, transthoracic echocardiography is routinely performed and reported by dedicated sonographers. Their training and accreditation fulfils most of the criteria set out by the ASA to perform TOE. We therefore considered a natural extension of their role would be to perform and interpret intraoperative TOEs.

Methods: Three experienced sonographers were trained in intraoperative TOEs by: (1) attending routine out-patient TOE lists and training courses, (2) individual teaching and supervision in live theatre cases by an experience cardiac radiologist, and (3) regular audit and review of studies and reports.

Results/Conclusions: All three sonographers now routinely perform and interpret intraoperative TOEs following the insertion of the transducer by the anaesthetist. This protocol has worked very successfully. Since the sonographer service started, there has been an increase of over $100 \%$ in the number of TOEs. Feedback from the cardiothoracic surgeons, anaesthetists, and sonographers has been very positive. At all times, clinical responsibility remains with the consultant in charge of the echocardiography department.

\section{The digital echocardiography department-a reality?}

R Keal, J Hayton, L Ricketts, S Waterfield; Department of Echocardiography, Glenfield Hospital, Groby Road, Leicester

Introduction: Traditionally, echocardiographic examinations have been recorded and stored in analogue format on VHS tape. This has a number of disadvantages, not least poor image quality and difficulty in retrieval. Modern systems come with digital storage as standard. Recent developments in computing power have now allowed the introduction of central digital archiving and image transmission. We describe our experiences in the development and introduction of a totally digital echocardiography department.

Method: As part of the upgrade of hospital facilities due to increased demand, the echocardiography department at Glenfield Hospital was allocated $£ 450000$ to purchase six echocardiography systems and an integrated digital archiving and reporting system. Following European tender, the contract was placed and the systems were installed in September 1999 . 
Results: The systems were installed in three days using the existing hospital network. Originally four months were allowed for the change from analogue to digital working, but the ease of use and major advantages of the system meant that the change over was completed within two weeks. Since then, over 5000 studies have been performed with the system and further expansion is planned. After initial minor problems, it has been extremely reliable and is now essential to the echocardiography department

Conclusion: The digital echocardiography department is now a practical reality and within a few years all departments will work this way. This paper will describe the problems and pitfalls encountered during the introduction of the system and describe the many benefits. 